\title{
Double Trouble-COVID-19 and the Widespread Use of Corticosteroids: Are We Staring at an Osteonecrosis Epidemic?
}

\author{
Gautam M. Shetty ${ }^{1,2}$ (D) \\ Received: 23 June 2021 / Accepted: 10 October 2021 / Published online: 26 October 2021 \\ (c) Indian Orthopaedics Association 2021
}

\begin{abstract}
Background A combination of immune-mediated vascular damage and routine use of systemic corticosteroid (CS) therapy in COVID-19 may significantly increase the risk and burden of osteonecrosis (ON) after COVID-19. This narrative review explores the pathogenesis, risk factors, and possible preventive and early treatment measures for ON in COVID-19.

Methods For this narrative review, an extensive literature search was performed using the PubMed, Medline, and Science Direct databases from January 2000 to August 2021 for relevant articles on etiopathogenesis, epidemiology, clinical manifestations, and treatment of severe acute respiratory syndrome coronavirus (SARS-CoV) infection and steroid-induced ON (SION).

Results Pathogenesis of COVID-19, utility of corticosteroids in the treatment of COVID-19, pathogenesis of SION vis-a-vis SARS-CoV infection, associated risk factors, and early diagnosis and treatment of ON following CS therapy of SARS-CoV infection were discussed.

Conclusion Preliminary data of COVID-19 and similar trends from the SARS 2003 epidemic indicate that the "angiocentric" pathogenesis of SARS-CoV-2 and treatment with high-dose CS may increase the risk of ON in COVID-19 patients. Risk stratification based on CS intake during COVID-19 treatment can help identify subjects at moderate to high-risk for ON where early preventive and follow-up plans can be initiated.
\end{abstract}

Keywords COVID-19 $\cdot$ Pandemic $\cdot$ Osteonecrosis $\cdot$ Corticosteroids $\cdot$ Avascular necrosis

\section{Introduction}

As of 31 August 2021, there were over 216.8 million confirmed cases of the severe acute respiratory syndrome coronavirus-2 (SARS-CoV-2) infection with over 4.5 million deaths globally due to the coronavirus disease 2019 (COVID-19) pandemic [1]. Currently, with subsequent waves of pandemic occurring in several countries, COVID19 may become endemic around the globe due to virus mutation and "immune escape", variations in immunity among populations, and uneven vaccine distribution [2].

Apart from a variety of symptoms, COVID-19 can cause musculoskeletal symptoms which include fatigue, myalgia,

Gautam M. Shetty

gautams10@gmail.com

1 Knee and Orthopaedic Clinic, 407B, Galleria, Hiranandani Gardens, Powai, Mumbai 400076, India

2 AIMD Research, Mumbai, India and arthralgia during the initial phase of the illness and myositis and rhabdomyolysis with severe illness [3, 4]. Clinical presentations of COVID-19 have been attributed to an immune-mediated vascular disease causing micro- and microvascular endothelial damage and hypercoagulability [5-8]. Systemic corticosteroid (CS) therapy is frequently administered to hospitalised patients with severe or critical COVID-19 and has been reported to improve survival and shorten the duration of hospitalisation [9-11]. Despite lack of clinical benefits and potential harmful effects when used in the early phase of COVID-19, CS therapy has also been widely used in non-critically ill patients with mild or moderate COVID-19 [12-14].

Steroid-induced osteonecrosis of the femoral head $(\mathrm{ONFH})$ is responsible for up to $47.4 \%$ of all patients diagnosed with non-traumatic ONFH $[15,16]$. The risk of steroid-induced ON (SION) depends on the cumulative and maximum dose and the underlying disease state $[15,17,18]$, and may occur even with low doses, especially in the presence of co-morbidities such as diabetes $[19,20]$. Although 
the occurrence of SION following treatment of severe acute respiratory syndrome (SARS) during the SARS 2003 epidemic has been well reported [21-29], few have called for attention regarding ON following COVID-19 [30-32].

Although the prevalence of ON in COVID-19 is unknown and may take time to reveal itself, it is crucial to understand the pathogenesis and risk factors for $\mathrm{ON}$ as a consequence of COVID-19 and its treatment. Furthermore, there is an urgent need to identify preventive and early diagnostic and treatment measures in COVID-19 patients to avert a possible epidemic of ON which may occur in the coming years. Hence this narrative review explores the pathogenesis, risk factors, and possible prevention and early treatment of ON in COVID-19.

\section{Literature Search}

This narrative review aimed to look at current knowledge about the pathogenesis of the COVID-19, utility of corticosteroids in the treatment of COVID-19, pathogenesis of SION vis-à-vis SARS-CoV infection (COVID-19 and SARS 2003), and the associated risk factors, early diagnosis and treatment of ON following CS therapy of SARS-CoV infection. An extensive literature search was performed using the PubMed, Medline, and Science Direct databases from January 2000 to August 2021. The search terms used included "SARS" OR “COVID-19" OR "coronavirus" OR "severe acute respiratory syndrome coronavirus 2", and "steroid" OR "corticosteroid" OR "glucocorticoid" OR "treatment", and "necrosis of the femoral head" OR "osteonecrosis" OR "avascular necrosis" OR "steroid-induced osteonecrosis", and "pathogenesis" OR "etiology". The inclusion criteria were all cohort and case-control studies, case series, case reports, review articles, and letters to the editor related to etiology, epidemiology, pathogenesis, clinical manifestations, or treatment of the SARS-CoV infection and SION. The exclusion criteria were non-English language articles and articles which were not peer-reviewed. Although articles published in the past 21 years were primarily selected, commonly referenced or cited older publications, especially related to SION, were also included for this review. In addition, the reference lists of articles identified by this search strategy were also manually searched and relevant articles were used. The etiology, epidemiology, pathogenesis, clinical manifestations, and treatment were reviewed from relevant articles related to COVID-19, SARS 2003, and SION obtained from the literature search.

\section{"Angiocentric" Pathogenesis of COVID-19}

The SARS-CoV-2 can directly infect endothelial cells using the angiotensin converting enzyme 2 (ACE2) receptor causing immune-mediated endothelial damage [5-8, 33-36]. Several researchers have proposed this "angiocentric" inflammation, and the ensuing endothelial dysfunction as central to the pathogenesis of COVID-19 [5-8, 33-36]. Furthermore, SARS-CoV-2 elicits a severe immune response leading to activation of the coagulation cascade and hypercoagulability [6, 7, 33-36]. The vascular changes in COVID-19 include endothilialitis, vascular narrowing and disruption, thrombotic microangiopathy, capillary dysfunction, and poor tissue oxygenation [6, 7, 33-36]. This combination of a hyperinflammatory and hypercoagulable state leads to widespread breakdown of vascular function in COVID-19 [6, 7, 33-36]. Prolonged vascular inflammation seen in COVID-19 may lead to increased vascular stiffness, accelerated vascular ageing, and increased long-term risk of cardiovascular disease [37, 38]. Furthermore, drugs such as hydroxychloroquine, azithromycin, and lopinavir used in treatment of COVID-19, can compromise cardiac function, especially in subjects with preexisting cardiovascular disease [39, 40]. Figure 1 outlines the pathogenesis of ON in COVID-19 through different pathways.

Although it primarily affects the lungs, extra-pulmonary manifestations are frequently seen in COVID-19 [41]. This could be due to the presence of ACE2 receptors in several extra-pulmonary tissues which may cause direct viral tissue damage, endothelial damage, and dysregulation of local immune responses [5, 41, 42]. The ACE2 receptor is locally expressed in skeletal muscles and bone marrow-derived stem/progenitor cells (BMSPCs) [43-45], and their targeting by SARS-CoV-2, may lead to sarcopenia, reduced muscle strength, altered skeletal repair, and anaphasic bone loss [43, 45-47]. The pathogenesis and progression of non-traumatic $\mathrm{ON}$ is multifactorial and involves a combination of various mechanisms such as hypercoagulability, suppression of angiogenesis, hyperadipogenesis, altered bone remodelling, and genetic predisposition [48-52]. Hence, the macro- and microvascular changes and tissue damage caused by SARSCoV-2 infection may increase the risk of ON after COVID-19. Although the vascular pathogenesis of ON after COVID-19 has not been investigated, a recent study reported significant vascular alteration in lower limbs with higher arterial stiffness, impaired femoral artery function, reduced ability of smaller arterioles to dilate with limb movements, and reactive hyperemia among young adults tested positive for SARS-CoV-2 


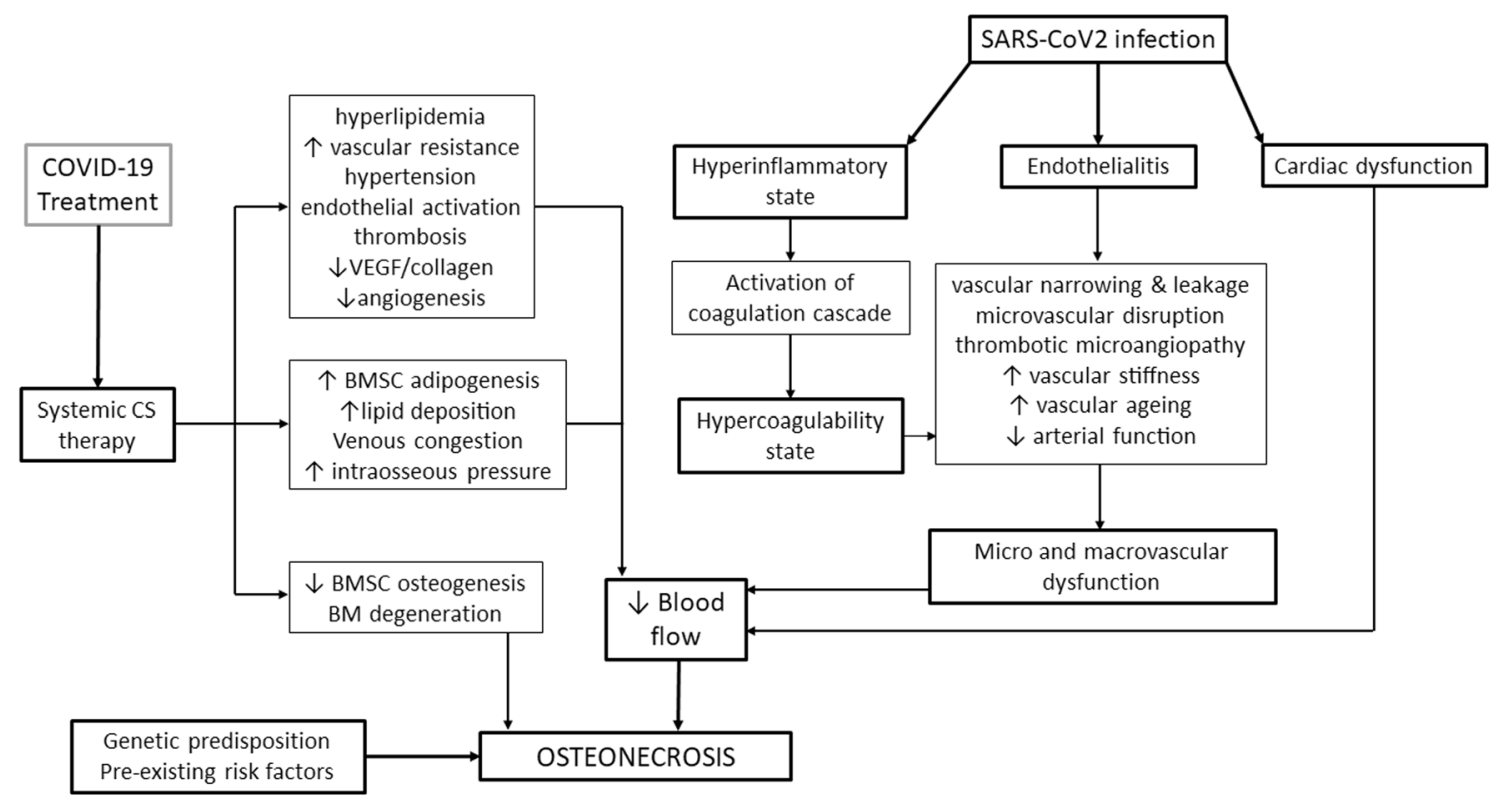

Fig. 1 Pathogenesis of osteonecrosis in COVID-19 through different pathways. COVID-19-coronavirus disease 2019; SARS-CoV-2severe acute respiratory syndrome coronavirus-2; CS — corticosteroid;
VGEF — vascular endothelial growth factor; BMSC-bone marrow stem cell; BM-bone matrix

severe COVID-19 pneumonia or acute respiratory distress syndrome (ARDS) [59, 60], in critically ill patients [61], and to prevent severe hypoxia in low resource settings [62]. Furthermore, CS therapy can cause significant systemic metabolic effects such as hyperglycemia, hyperlipidemia, and hypertension, reduced bone matrix formation, and increased bone resorption [63].

SION have been reported to occur with moderate doses administered over a long duration ( $>20 \mathrm{mg} /$ day of prednisolone) $[64,65]$, or with high doses (1-2gm/day) over a short duration $[17,66]$. Studies have analysed the incidence of ON after CS treatment following the SARS 2003 epidemic [21]. Zhao et al. [21] in a meta-analysis of patients with SARS 2003, reported a SION prevalence of $26.6 \%$ (303/1137 patients) at a mean follow-up of 15.1 months (2.5-90 months), and that higher cumulative dose (relative risk of 1.57 per $5 \mathrm{~g}$ increase) and longer treatment duration (relative risk of 1.29 for each 10-day increment) increased the risk of SION. Furthermore, a peak dose of $>200 \mathrm{mg}$ or a cumulative dose of $>4000 \mathrm{mg}$ of MPS may increase the risk of multifocal (epiphyseal and diaphyseal) ON lesions [67].

The pathogenesis of SION is complex and multifactorial. Corticosteroids diminish local perfusion by inducing microvascular thrombosis and increasing intraosseous pressure by promoting intramedullary adipogenesis which causes arterial obstruction and venous stasis [15, 40, 68]. Corticosteroids also cause an imbalance in bone resorption and repair by decreasing osteoblast production, increasing osteocyte apoptosis, and prolonging osteoclast lifespan [15, 
40] (Fig. 1). The presence of co-morbidities such as alcohol consumption [69], smoking [70], cardiovascular or cerebrovascular disease [71], may further accentuate the effect of $\mathrm{CS}$ in causing $\mathrm{ON}$.

\section{Risk Factors for ON in COVID-19}

Data are currently lacking on the relationship between cumulative CS dosage and ON after COVID-19. However, analysis of clinical studies which reported ON following CS therapy for SARS 2003 indicated an ON prevalence rate of $5-57.7 \%$ and the association of higher cumulative CS dose and longer duration of CS therapy with greater risk of ON (Table 2). A meta-analysis of patients from the SARS 2003 epidemic, reported that a higher cumulative dose (> $5000 \mathrm{mg}$ MPS-equivalent dose) and longer treatment duration ( $>10$ days) increased the risk of SION [21]. A recent review article by Zhang et al. [30] analysed the risk of ONFH with CS treatment of COVID-19 by juxtaposing it with the experience and lessons learned from CS therapy used during the SARS 2003 epidemic. Based on data derived from the SARS 2003 epidemic, the authors suggested that a cumulative dose of $<5000 \mathrm{mg}$ of MPS may be relatively safe and the risk of $\mathrm{ON}$ increased with increasing cumulative doses, with the highest risk when the total dose was about
10,000-15,000 mg of MPS [30]. However, a recent case series of three patients who developed ONFH after COVID19 [32], reported a lower mean cumulative dose of $758 \mathrm{mg}$ (400-1250 mg) MPS in their patients, which was less than the mean cumulative dose of around $2000 \mathrm{mg}$ documented in the literature as causative for SION $[64,66]$, and for ONFH following the SARS 2003 disease (Table 2).

Although patients with COVID-19 who received higher cumulative dose and longer duration of CS may be at greater risk for ON, it may not be possible to know the actual dosage and duration of CS treatment in every patient. Hence patient history about clinical course of COVID-19 may provide important clues regarding higher dosage and longer duration of CS treatment and help identify "COVID-related" risk factors (Table 1). These include severe organised COVID19 pneumonia, moderate to severe ARDS, and significantly elevated serum markers where high dose CS are frequently used [58, 59, 72]. Similarly, presence of co-morbidities may indicate pre-existing risk factors where even low dose CS may increase the risk of ON (Table 1).

A risk stratification system for SION in COVID-19 patients has been proposed by Zhang and Zhang [31] to identify patients with low-risk (not received CS); moderate-risk (received cumulative dose of $<2000 \mathrm{mg}$ CS for $<1$ week); and high-risk (received cumulative dose of $\geq 2000 \mathrm{mg} \mathrm{CS}$ for $\geq 1$ week or IV pulse $\geq 80 \mathrm{mg} /$ day for at least 3 days).
Table 1 Potential risk factors for osteonecrosis after COVID infection

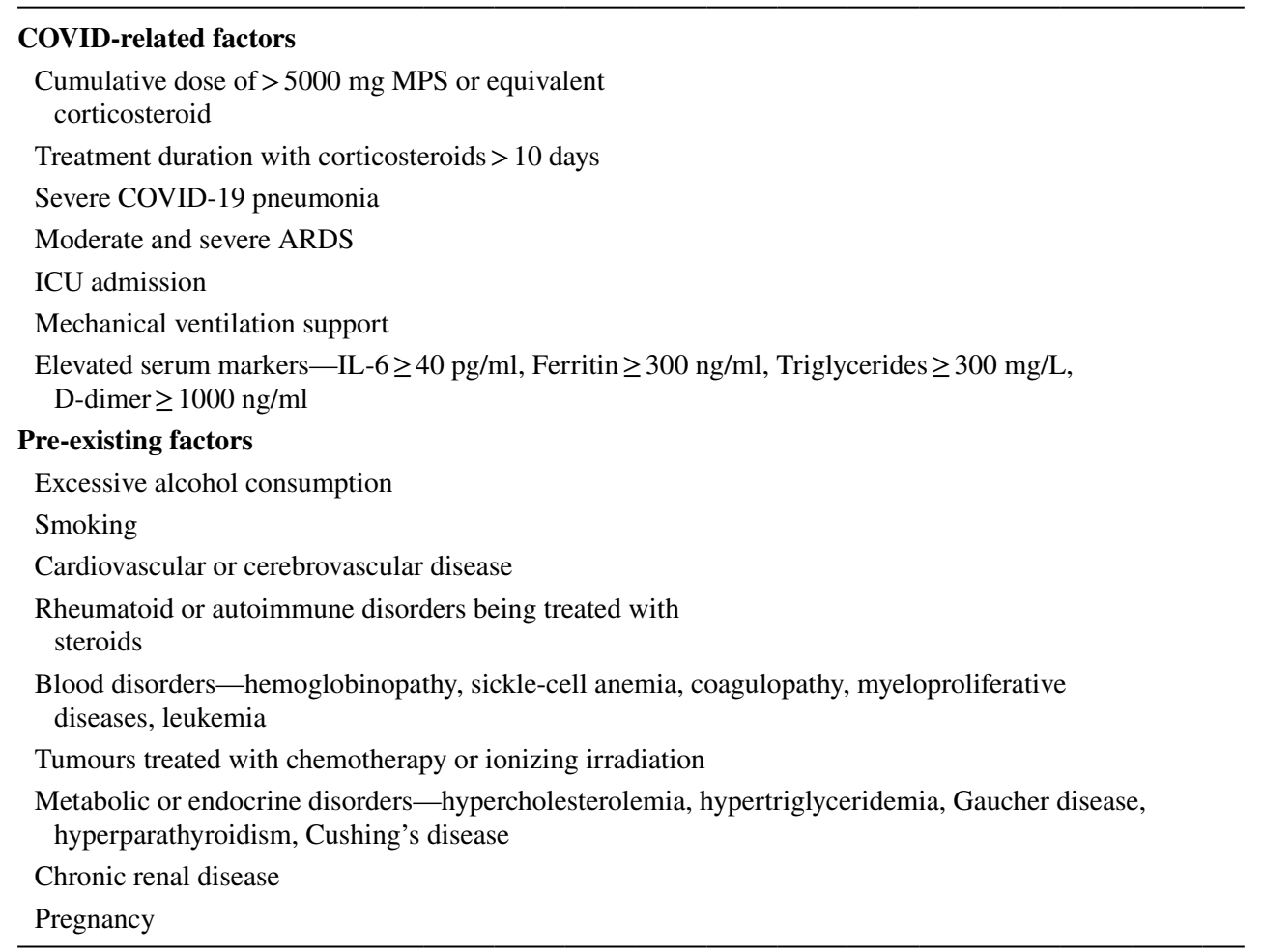

COVID-19 coronavirus disease 2019, ARDS acute respiratory distress syndrome, ICU intensive care unit, $I L$ interlukin, MPS methylprednisolone 
230

Indian Journal of Orthopaedics (2022) 56:226-236

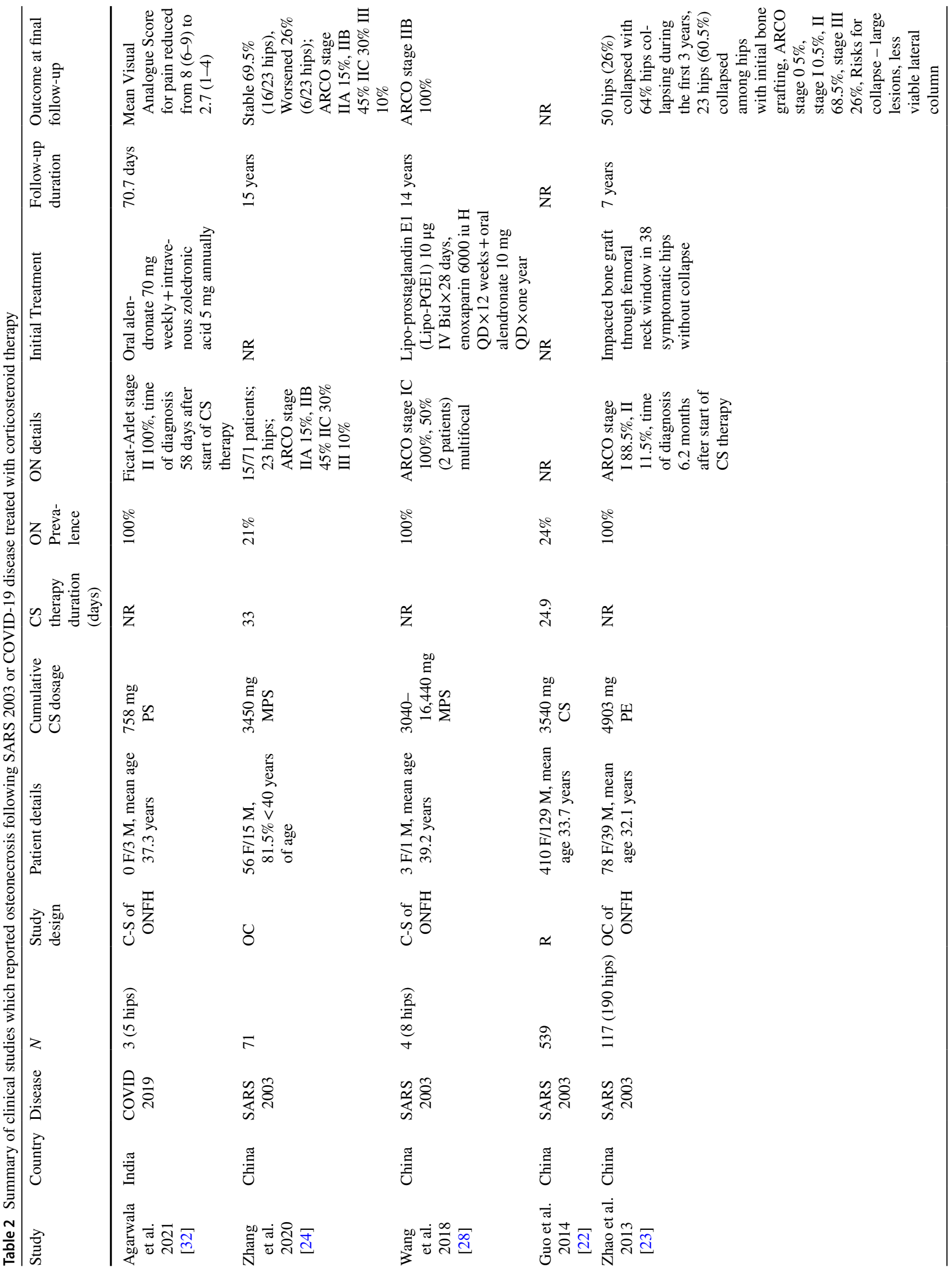

Springer 
Indian Journal of Orthopaedics (2022) 56:226-236

231

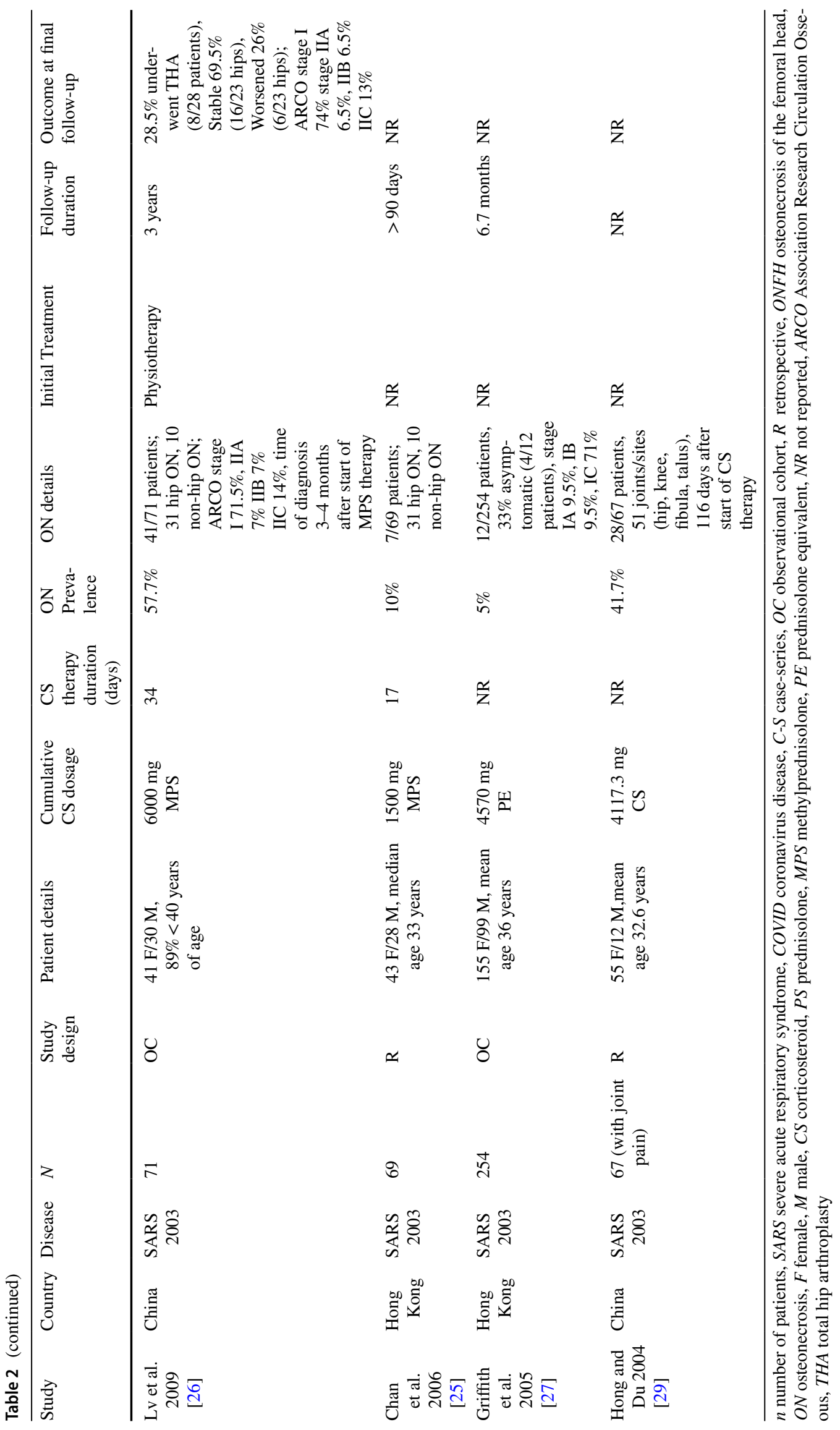

Springer 
However, pre-existing risk factors should be factored in for risk stratification of ON in COVID-19 patients as their existence may cause SION even in lower cumulative doses of CS. Furthermore, the inherent "angiocentric" nature of the COVID-19 disease and its effect on limb vascularity $[53,54]$, may increase the risk of ON even in patients who did not receive CS therapy for COVID-19. As more clinical data become available in the coming years on the mid to long term effects of COVID-19, evidence will emerge if COVID-19 can increase the risk of ON even in the absence of CS therapy. Hence, this risk stratification system may need further modification and validation as more evidence emerges in the future.

\section{Prevention, Early Diagnosis and Treatment of ON in COVID-19}

As part of prevention, judicious use of CS with a clear indication during COVID-19 treatment should be followed. A cumulative MPS-equivalent dose of $<5000 \mathrm{mg}$ and course duration $<10$ days may lower the risk of ON [21]. This cumulative dose and duration may have to be further reduced in subjects with pre-existing risk factors for ON. However, since CS therapy may be life-saving and can potentially reduce morbidity, caution is urged on withholding, withdrawing, or using a suboptimal dose of CS therapy in critically ill patients with COVID-19 who might otherwise benefit from it. Methods to effectively prevent the development of SION or progression of early disease are unclear with most studies performed on animal models [15]. Intermittent rather than continuous CS treatment with a "steroid holiday" [73], and addition of lipid-lowering statins and anti-coagulants with or without a vasodilator [74], may help reduce the risk of ON. Despite encouraging results in animal models [74, 75], the efficacy of lipid lowering statins in preventing SION in human subjects is unclear [76]. However, anti-coagulants such as low-molecular weight heparin (LMWH) or enoxaparin may prevent onset and progression of SION [77-79]. Although anticoagulants are frequently administered in severe COVID-19 to prevent or treat thromboembolic complications [80], there may be a need to continue standard thromboprophylaxis in high-risk patients to prevent $\mathrm{ON}$.

Early diagnosis and timely treatment of $\mathrm{ON}$ is necessary as many patients who undergo CS treatment for COVID-19 are young individuals in whom joint-salvaging treatment options for advanced disease are limited. Magnetic resonance imaging (MRI) evidence of ON can develop within 1 year after initiation of high dose CS therapy [21, 81, 82]. However, the interval between corticosteroid intake and development of symptomatic ON appears to be shorter (around 2-6 months) in patients with SARS-CoV infection
(COVID-19 or SARS 2003 disease) (Table. 2), when compared to the 6-12 months reported for non-SARS-CoV SION [64-67]. Hence it is important to remain vigilant for the possibility of development of ON during the first year in high-risk patients after COVID-19 treatment. Clinical symptoms appear much later than radiological changes in ONFH, and are often misdiagnosed as originating from the lumbar spine or the knee joint [21, 82]. Hence, regular hip monitoring using MRI should be carried out in high-risk patients, at 3,6, and 12 months after initiation of CS treatment for COVID-19 [82]. Apart from MRI, serum biomarkers such as serum apolipoprotein A-IV, human cartilage glycoprotein-39, interleukin 33, adiponectin, and antiphospholipid antibodies may be useful for early diagnosis of ON [83-85].

Early treatment of SION may help delay disease progression, reduce morbidity, and the risk for joint replacement surgery in such patients. Intervention depends on the stage of disease and includes bisphosphonates (oral alendronate $70 \mathrm{mg}$ weekly along with IV zoledronic acid $5 \mathrm{mg}$ annually) [32], or anticoagulants (low-molecularweight heparin 5000LXU subcutaneously, once daily for 2 weeks every 6 months) with limited weight-bearing [78] for stage I disease, and simple core decompression for stage II disease [78]. Fu et al. [78], in a study of early diagnosis and treatment of 60 patients with ONFH reported no progression of disease in $76.3 \%$ of patients with stage I disease treated with LMWH and in $80 \%$ of patients with stage II disease treated with core decompression at the end of 24 months. Zhao et al. [23], in an analysis of 38 symptomatic ON hips without collapse diagnosed at a mean 6.2 months after start of CS therapy for SARS 2003 disease and treated with impacted bone grafting through femoral neck window, reported femoral head collapse among $60.5 \%$ of these hips at the end of 7 years (Table. 2). Wang et al. [28], in a case-series of four hips with ON following CS therapy for SARS 2003 disease and treated with a combination of IV Lipo-prostaglandin E1 $10 \mu \mathrm{g}$ Bid for 4 weeks, enoxaparin $6000 \mathrm{IU}$ $\mathrm{H}$ QD for 12 weeks, and oral alendronate $10 \mathrm{mg}$ QD for 1 year, reported slow progression of ONFH lesion from IC to IIB with no change in Harris hip score at the end of 14 years. Although literature is lacking on the results of early treatment of ON after COVID infection, a recent case series of three patients who developed ONFH after a mean 58 days post COVID-19 diagnosis, reported significant improvement in pain after bisphosphonate therapy at a mean follow-up of 70 days [32]. Traditional Chinese herbal medicines (e.g., Huo-Gu formula) given for 6 months after the diagnosis of ONFH in SARS 2003 patients have been reported to prevent femoral head collapse, delay replacement surgery, and maintain physical function in the long term [86]. 


\section{Limitations}

The pathophysiological mechanisms of ON in COVID-19, described in the current review, were derived from the pathogenesis of COVID-19 and SION related to SARS 2003 previously described in the literature. Hence these need to be further validated with in vitro, in vivo, and clinical studies. As data on ON following COVID-19 disease are scarce, most articles used in this narrative review regarding prevalence, risk factors, early diagnosis, and treatment of ON were based on previous studies of the SARS 2003 epidemic. Hence, the consequences of CS therapy in patients with COVID-19 disease and the effect of early diagnosis and treatment of ON in COVID-19 need confirmation by future clinical studies. Despite these limitations, this article is an early and much-needed attempt to understand the pathogenesis, risk factors, diagnosis, and treatment of ON as a consequence of COVID-19 and helps identify gaps in knowledge about this significant consequence of COVID-19.

\section{Recommendations}

Clinical data from the SARS 2003 epidemic indicate a greater propensity to develop ON in patients after SARS$\mathrm{CoV}$ infection treated with CS. Hence, corticosteroids may have to be administered with caution, including minimizing dose and duration where feasible, and avoiding multiple types of CS for COVID-19 treatment. These include limiting cumulative MPS-equivalent dose to $<5000 \mathrm{mg}$ and the total duration of CS therapy to $<10$ days. With increasing number of studies being published on the efficacy of various treatment methods for COVID-19, alternatives to CS to alleviate hyperinflammation, and reduce morbidity and mortality, with a better safety profile and reduced risk of ON, may need to be explored.

Based on a risk-stratification system for ON in COVID19, similar to the one recommended by Zhang and Zhang [31], moderate-risk (who received a cumulative dose of $<2000 \mathrm{mg}$ CS for $<1$ week) and high-risk (who received cumulative dose of $\geq 2000 \mathrm{mg}$ CS for $\geq 1$ week or IV pulse $\geq 80 \mathrm{mg} /$ day for at least 3 days) subjects with COVID-19 disease may have to be prescribed specific preventive and follow-up plans on discharge [31]. These include pharmacological agents such as bisphosphonates, anticoagulants, and vasodilators which can be prescribed in moderate to high-risk subjects during COVID-19 treatment and continued after discharge to prevent ON. Regular follow-up with MRI imaging (e.g., at 3, 6, and 12 months after initiation of CS treatment for COVID-19) during the first 1-3 years after COVID-19 can help in early detection of $\mathrm{ON}$ in moderate to high-risk patients. A combination of physical therapy and pharmacotherapy (bisphosphonates, anticoagulants, and vasodilators) can be used to delay progression of early stage $\mathrm{ON}$.

\section{Conclusions}

With successive "waves" of the COVID-19 pandemic, patients infected with SARS-CoV-2 who receive CS treatment will increase substantially in the coming years. Preliminary data of COVID-19 and similar trends from the SARS 2003 epidemic indicate that the "angiocentric" pathogenesis of SARS-CoV-2 and treatment with high-dose CS may increase the risk of ON in COVID19 patients. Hence, healthcare systems around the world may face a significant increase in $\mathrm{ON}$ cases among the general population in the near future. Risk stratification based on CS intake during COVID-19 treatment can help identify subjects at moderate to high risk for ON where early preventive and follow-up plans can be initiated. Evidence regarding musculoskeletal manifestations and long-term consequences of COVID-19 and its treatment with CS continue to evolve. It is currently based on observational studies, consensus statements, expert opinion, studies from the SARS 2003 epidemic and high-quality evidence is lacking. Moving ahead, research needs to focus on in vivo and clinical studies to determine the risk and pathogenesis of ON after COVID-19 and the efficacy of various preventive and treatment modalities in these patients.

Funding No benefits or funds were received in support of this study by any of the authors.

\section{Declarations}

Conflict of Interest The author declares that he has no conflict of interest.

Ethical Standard Statement This article does not contain any studies with human or animal subjects performed by the any of the authors.

Ethics and Institutional Review Board (IRB) Approval Not required.

Informed consent For this type of study, formal consent is not required. 


\section{References}

1. No authors listed. WHO Coronavirus (COVID-19) Dashboard. https://covid19.who.int/. Accessed 01 Sep 2021

2. Phillips, N. (2021). The coronavirus is here to stay-here's what that means. Nature, 590(7846), 382-384. https://doi.org/10.1038/ d41586-021-00396-2

3. Mehta, O. P., Bhandari, P., Raut, A., Kacimi, S. E. O., \& Huy, N. T. (2021). Coronavirus disease (COVID-19): comprehensive review of clinical presentation. Frontiers of Public Health. https:// doi.org/10.3389/fpubh.2020.582932

4. Cipollaro, L., Giordano, L., Padulo, J., Oliva, F., \& Maffulli, N. (2020). Musculoskeletal symptoms in SARS-CoV-2 (COVID-19) patients. Journal of Orthopedic Surgery and Research, 15(1), 178. https://doi.org/10.1186/s13018-020-01702-w

5. Ramos-Casals, M., Brito-Zerón, P., \& Mariette, X. (2021). Systemic and organ-specific immune-related manifestations of COVID-19. Nature Reviews Rheumatology, 17(6), 315-332. https://doi.org/10.1038/s41584-021-00608-z

6. Siddiqi, H. K., Libby, P., \& Ridker, P. M. (2021). COVID-19-a vascular disease. Trends in Cardiovascular Medicine, 31(1), 1-5. https://doi.org/10.1016/j.tcm.2020.10.005

7. Østergaard, L. (2021). SARS CoV-2 related microvascular damage and symptoms during and after COVID-19: Consequences of capillary transit-time changes, tissue hypoxia and inflammation. Physiological Reports. https://doi.org/10.14814/phy2.14726

8. Osuchowski, M. F., Winkler, M. S., Skirecki, T., et al. (2021). The COVID-19 puzzle: deciphering pathophysiology and phenotypes of a new disease entity. The Lancet, 9(6), 622-642. https://doi.org/ 10.1016/S2213-2600(21)00218-6

9. van Paassen, J., Vos, J. S., Hoekstra, E. M., Neumann, K. M. I., Boot, P. C., \& Arbous, S. M. (2020). Corticosteroid use in COVID-19 patients: a systematic review and meta-analysis on clinical outcomes. Critical Care, 24(1), 696. https://doi.org/10. 1186/s13054-020-03400-9

10. Cano, E. J., Fonseca Fuentes, X., Corsini Campioli, C., et al. (2021). Impact of corticosteroids in coronavirus disease 2019 outcomes: systematic review and meta-analysis. Chest, 159(3), 1019-1040. https://doi.org/10.1016/j.chest.2020.10.054

11. RECOVERY Collaborative Group, Horby, P., Lim, W. S., et al. (2021). Dexamethasone in Hospitalized Patients with Covid-19. New England Journal of Medicine, 384(8), 693-704. https://doi. org/10.1056/NEJMoa2021436

12. Shuto, H., Komiya, K., Yamasue, M., et al. (2020). A systematic review of corticosteroid treatment for noncritically ill patients with COVID-19. Scientific Reports, 10(1), 20935. https://doi.org/ 10.1038/s41598-020-78054-2

13. Romano, G. M., Cafiero, T., Frangiosa, A., \& Robertis, E. (2021). Corticosteroids in patients with COVID-19, use and misuse: a brief review. Minerva Anestesiologica. https://doi.org/10.2373/ S0375-9393.21.15625-1

14. Zhan, Y., Shang, J., Gu, Y., Huang, Q., \& Xie, J. (2021). Efficacy of corticosteroid in patients with COVID-19: a multi-center retrospective study and meta-analysis. Journal of Medical Virology, 93(7), 4292-4302. https://doi.org/10.1002/jmv.26914

15. Xie, X. H., Wang, X. L., Yang, H. L., Zhao, D. W., \& Qin, L. (2015). Steroid-associated osteonecrosis: epidemiology, pathophysiology, animal model, prevention, and potential treatments (an overview). Journal of Orthopaedic Translation, 3(2), 58-70. https://doi.org/10.1016/j.jot.2014.12.002

16. Ikeuchi, K., Hasegawa, Y., Seki, T., Takegami, Y., Amano, T., \& Ishiguro, N. (2015). Epidemiology of nontraumatic osteonecrosis of the femoral head in Japan. Modern Rheumatology, 25(2), 278-281. https://doi.org/10.3109/14397595.2014.932038
17. Powell, C., Chang, C., Naguwa, S. M., Cheema, G., \& Gershwin, M. E. (2010). Steroid induced osteonecrosis: an analysis of steroid dosing risk. Autoimmune Reviews, 9(11), 721-743. https://doi.org/ 10.1016/j.autrev.2010.06.007

18. Lai, S. W., Lin, C. L., \& Liao, K. F. (2020). Evaluating the association between avascular necrosis of femoral head and oral corticosteroids use in Taiwan. Medicine (Baltimore). https://doi.org/ 10.1097/MD.0000000000018585

19. Rajput, S., \& Kulshreshtha, B. (2018). Bilateral femoral head avascular necrosis with physiological doses of steroids. Indian Journal of Endocrinology and Metabolism, 22(5), 710-711. https://doi.org/10.4103/ijem.IJEM_97_18

20. Lai, S. W., Lin, C. L., \& Liao, K. F. (2019). Real-world database examining the association between avascular necrosis of the femoral head and diabetes in Taiwan. Diabetes Care, 42(1), 39-43. https://doi.org/10.2337/dc18-1258

21. Zhao, R., Wang, H., Wang, X., \& Feng, F. (2017). Steroid therapy and the risk of osteonecrosis in SARS patients: a dose-response meta-analysis. Osteoporosis International, 28(3), 1027-1034. https://doi.org/10.1007/s00198-016-3824-z

22. Guo, K. J., Zhao, F. C., Guo, Y., Li, F. L., Zhu, L., \& Zheng, W. (2014). The influence of age, gender and treatment with steroids on the incidence of osteonecrosis of the femoral head during the management of severe acute respiratory syndrome: a retrospective study. Bone and Joint Journal, 96-B(2), 259-262. https://doi.org/ 10.1302/0301-620X.96B2.31935

23. Zhao, F. C., Guo, K. J., \& Li, Z. R. (2013). Osteonecrosis of the femoral head in SARS patients: seven years later. European Journal of Orthopaedic Surgery and Traumatology, 23(6), 671-677. https://doi.org/10.1007/s00590-012-1054-4

24. Zhang, P., Li, J., Liu, H., et al. (2020). Long-term bone and lung consequences associated with hospital-acquired severe acute respiratory syndrome: a 15 -year follow-up from a prospective cohort study. Bone Research, 8, 1-8. https://doi.org/10.1038/ s41413-020-0084-5

25. Chan, M. H., Chan, P. K., Griffith, J. F., et al. (2006). Steroidinduced osteonecrosis in severe acute respiratory syndrome: a retrospective analysis of biochemical markers of bone metabolism and corticosteroid therapy. Pathology, 38(3), 229-235. https://doi.org/10.1080/00313020600696231

26. Lv, H., de Vlas, S. J., Liu, W., et al. (2009). Avascular osteonecrosis after treatment of SARS: a 3-year longitudinal study. Tropical Medicine and International Health, 14(Suppl 1), 79-84. https://doi.org/10.1111/j.1365-3156.2008.02187.x

27. Griffith, J. F., Antonio, G. E., Kumta, S. M., et al. (2005). Osteonecrosis of hip and knee in patients with severe acute respiratory syndrome treated with steroids. Radiology, 235(1), 168-175. https://doi.org/10.1148/radiol.2351040100

28. Wang, W., Zhang, N., Guo, W., \& Gao, F. (2018). Combined pharmacotherapy for osteonecrosis of the femoral head after severe acute respiratory syndrome and interstitial pneumonia: two and a half to fourteen year follow-up. International Orthopaedics, 42(7), 1551-1556. https://doi.org/10.1007/ s00264-018-3907-x

29. Hong, N., \& Du, X. K. (2004). Avascular necrosis of bone in severe acute respiratory syndrome. Clinical Radiology, 59(7), 602-608. https://doi.org/10.1016/j.crad.2003.12.008

30. Zhang, S., Wang, C., Shi, L., \& Xue, Q. (2021). Beware of steroid-induced avascular necrosis of the femoral head in the treatment of COVID-19-experience and lessons from the SARS epidemic. Drug Design, Development and Therapy, 15, 983995. https://doi.org/10.2147/DDDT.S298691

31. Zhang, B., \& Zhang, S. (2020). Corticosteroid-induced osteonecrosis in COVID-19: a call for caution. Journal of Bone and Mineral Research, 35(9), 1828-1829. https://doi.org/10.1002/ jbmr.4136 
32. Agarwala, S. R., Vijayvargiya, M., \& Pandey, P. (2021). Avascular necrosis as a part of "long COVID-19." BML Case Reports. https://doi.org/10.1136/bcr-2021-242101

33. Ackermann, M., Mentzer, S. J., Kolb, M., \& Jonigk, D. (2020). Inflammation and intussusceptive angiogenesis in COVID19: everything in and out of flow. The European Respiratory Journal, 56(5), 2003147. https://doi.org/10.1183/13993003. 03147-2020

34. Quinaglia, T., Shabani, M., Breder, I., Silber, H. A., Lima, J. A. C., \& Sposito, A. C. (2021). Coronavirus disease-19: the multi-level, multi-faceted vasculopathy. Atherosclerosis, 322, 39-50. https:// doi.org/10.1016/j.atherosclerosis.2021.02.009

35. Bernard, I., Limonta, D., Mahal, L. K., \& Hobman, T. C. (2020). Endothelium infection and dysregulation by SARS-CoV-2: evidence and caveats in COVID-19. Viruses, 13(1), 29. https://doi. org/10.3390/v13010029

36. Barbosa, L. C., Gonçalves, T. L., de Araujo, L. P., Rosario, L. V. O., \& Ferrer, V. P. (2021). Endothelial cells and SARS-CoV-2: an intimate relationship. Vascular Pharmacology. https://doi.org/10. 1016/j.vph.2021.106829

37. Zanoli, L., Briet, M., Empana, J. P., et al. (2020). Vascular consequences of inflammation: a position statement from the ESH Working Group on Vascular Structure and Function and the ARTERY Society. Journal of Hypertension, 38(9), 1682-1698. https://doi.org/10.1097/HJH.0000000000002508

38. Bruno, R. M., Spronck, B., Hametner, B., et al. (2021). Covid19 effects on ARTErial stiffness and vascular AgeiNg: CARTESIAN study rationale and protocol. Artery Research, 27(2), 59-68. https://doi.org/10.2991/artres.k.201124.001

39. Nishiga, M., Wang, D. W., Han, Y., Lewis, D. B., \& Wu, J. C. (2020). COVID-19 and cardiovascular disease: from basic mechanisms to clinical perspectives. Nature Reviews Cardiology, 17(9), 543-558. https://doi.org/10.1038/s41569-020-0413-9

40. Pellicori, P., Doolub, G., Wong, C. M., et al. (2021). COVID-19 and its cardiovascular effects: a systematic review of prevalence studies. The Cochrane Database of Systematic Reviews. https:// doi.org/10.1002/14651858.CD013879

41. Gupta, A., Madhavan, M. V., Sehgal, K., et al. (2020). Extrapulmonary manifestations of COVID-19. Nature Medicine, 26(7), 1017-1032. https://doi.org/10.1038/s41591-020-0968-3

42. Fahmy, O. H., Daas, F. M., Salunkhe, V., et al. (2021). is microthrombosis the main pathology in coronavirus disease 2019 severity? - a systematic review of the postmortem pathologic findings. Critical Care Explorations. https://doi.org/10.1097/ CCE.0000000000000427

43. Yamamoto, K., Takeshita, H., \& Rakugi, H. (2020). ACE2, angiotensin 1-7 and skeletal muscle: review in the era of COVID-19. Clinical Science (London), 134(22), 3047-3062. https://doi.org/10.1042/CS20200486

44. Joshi, S., Balasubramanian, N., Vasam, G., \& Jarajapu, Y. P. (2016). Angiotensin converting enzyme versus angiotensin converting enzyme-2 selectivity of MLN-4760 and DX600 in human and murine bone marrow-derived cells. European Journal of Pharmacology, 774, 25-33. https://doi.org/10.1016/j. ejphar.2016.01.007

45. Tao, H., Bai, J., Zhang, W., et al. (2020). Bone biology and COVID-19 infection: Is ACE2 a potential influence factor? Medical Hypotheses. https://doi.org/10.1016/j.mehy.2020. 110178

46. Tanriverdi, A., Savci, S., Kahraman, B. O., \& Ozpelit, E. (2021). Extrapulmonary features of post-COVID-19 patients: muscle function, physical activity, mood, and sleep quality. Irish Journal of Medical Science, 6, 1-7. https://doi.org/10.1007/ s11845-021-02667-3

47. Tuzun, S., Keles, A., Okutan, D., Yildiran, T., \& Palamar, D. (2021). Assessment of musculoskeletal pain, fatigue and grip strength in hospitalized patients with COVID-19. European Journal of Physical Rehabilitation Medicine. https://doi.org/10.2373/ S1973-9087.20.06563-6

48. Mont, M. A., Salem, H. S., Piuzzi, N. S., Goodman, S. B., \& Jones, L. C. (2020). Nontraumatic osteonecrosis of the femoral head: where do we stand today? a 5-year update. Journal of Bone and Joint Surgery American, 102(12), 1084-1099. https://doi.org/ 10.2106/JBJS.19.01271

49. Kerachian, M. A., Harvey, E. J., Cournoyer, D., Chow, T. Y., \& Séguin, C. (2006). Avascular necrosis of the femoral head: vascular hypotheses. Endothelium, 13(4), 237-244. https://doi.org/10. 1080/10623320600904211

50. Pouya, F., \& Kerachian, M. A. (2015). Avascular necrosis of the femoral head: are any genes involved? The Archives of Bone and Joint Surgery, 3(3), 149-155.

51. Naik, A. A., Narayanan, A., Khanchandani, P., et al. (2020). Systems analysis of avascular necrosis of femoral head using integrative data analysis and literature mining delineates pathways associated with disease. Scientific Reports, 10(1), 18099. https:// doi.org/10.1038/s41598-020-75197-0

52. Petek, D., Hannouche, D., \& Suva, D. (2019). Osteonecrosis of the femoral head: pathophysiology and current concepts of treatment. EFORT Open Reviews, 4(3), 85-97. https://doi.org/10.1302/ 2058-5241.4.180036

53. Ratchford, S. M., Stickford, J. L., Province, V. M., et al. (2021) Vascular alterations among young adults with SARS-CoV-2. American Journal of Physiology, 320(1), H404-H410. https:// doi.org/10.1152/ajpheart.00897.2020

54. Goldman, I. A., Ye, K., \& Scheinfeld, M. H. (2020). Lowerextremity arterial thrombosis associated with COVID-19 is characterized by greater thrombus burden and increased rate of amputation and death. Radiology, 297(2), E263-E269. https://doi.org/ 10.1148/radiol.2020202348

55. Akbas, E. M., \& Akbas, N. (2021). COVID-19, adrenal gland, glucocorticoids, and adrenal insufficiency. Biomedical Papers of the Medical Faculty of the University Palacký, Olomouc, Czechoslovakia, 165(1), 1-7. https://doi.org/10.5507/bp.2021.011

56. Monreal, E., Sainz de la Maza, S., Natera-Villalba, E., et al. (2021). High versus standard doses of corticosteroids in severe COVID-19: a retrospective cohort study. European Journal of Clinical Microbiology \& Infectious Diseases, 40(4), 761-769. https://doi.org/10.1007/s10096-020-04078-1

57. Bahl, A., Johnson, S., \& Chen, N. W. (2021). Timing of corticosteroids impacts mortality in hospitalized COVID-19 patients. Internal and Emergency Medicine. https://doi.org/10.1007/ s11739-021-02655-6

58. Li, S., Hu, Z., \& Song, X. (2021). High-dose but not low-dose corticosteroids potentially delay viral shedding of patients with COVID-19. Clinical Infectious Diseases, 72(7), 1297-1298. https://doi.org/10.1093/cid/ciaa829

59. Pinzón, M. A., Ortiz, S., Holguín, H., et al. (2021). Dexamethasone vs methylprednisolone high dose for Covid-19 pneumonia. PLoS ONE. https://doi.org/10.1371/journal.pone.0252057

60. So, C., Ro, S., Murakami, M., Imai, R., \& Jinta, T. (2020). Highdose, short-term corticosteroids for ARDS caused by COVID-19: a case series. Respirology Case Reports, 8(6), e00596. https://doi. org/10.1371/journal.pone.0252057

61. WHO Rapid Evidence Appraisal for COVID-19 Therapies (REACT) Working Group, Sterne, J. A. C., Murthy, S., et al. (2020). Association between administration of systemic corticosteroids and mortality among critically ill patients with covid-19: a meta-analysis. JAMA, 324(13), 1330-1341. https://doi.org/10. 1001/jama.2020.17023

62. Edara, L., Suvvari, T. K., \& Kutikuppala, L. V. S. (2020). High dose steroid therapy to prevent severe hypoxia in COVID-19 
patients: a potential solution for low resource clinical setting. Cureus. https://doi.org/10.7759/cureus.12330

63. Alessi, J., de Oliveira, G. B., Schaan, B. D., \& Telo, G. H. (2020). Dexamethasone in the era of COVID-19: friend or foe? An essay on the effects of dexamethasone and the potential risks of its inadvertent use in patients with diabetes. Diabetology \& Metabolic Syndrome, 12, 80. https://doi.org/10.1186/s13098-020-00583-7

64. Aaron, R. K., Voisinet, A., Racine, J., Ali, Y., \& Feller, E. R. (2011). Corticosteroid-associated avascular necrosis: dose relationships and early diagnosis. Annals of the New York Academy of Sciences, 1240, 38-46. https://doi.org/10.1111/j.1749-6632.2011. 06218.x

65. Ono, K., Tohjima, T., \& Komazawa, T. (1992). Risk factors of avascular necrosis of the femoral head in patients with systemic lupus erythematosus under high-dose corticosteroid therapy. Clinical Orthopaedic and Related Research, 277(4), 89-97.

66. Arbab, D., \& König, D. P. (2016). Atraumatic femoral head necrosis in adults. Deutsches Ärzteblatt International, 113(3), 31-38. https://doi.org/10.3238/arztebl.2016.0031

67. Zhang, N. F., Li, Z. R., Wei, H. Y., Liu, Z. H., \& Hernigou, P. (2008). Steroid-induced osteonecrosis: the number of lesions is related to the dosage. Journal of Bone and Joint Surgery British, 90(9), 1239-1243. https://doi.org/10.1302/0301-620X.90B9. 20056

68. Wang, A., Ren, M., \& Wang, J. (2018). The pathogenesis of steroid-induced osteonecrosis of the femoral head: a systematic review of the literature. Gene, 671, 103-109. https://doi.org/10. 1016/j.gene.2018.05.091

69. Tsai, S. W., Wu, P. K., Chen, C. F., et al. (2016). Etiologies and outcome of osteonecrosis of the femoral head: etiology and outcome study in a Taiwan population. Journal of Chinese Medical Association, 79(1), 39-45. https://doi.org/10.1016/j.jcma.2015. 07.010

70. Wen, Z., Lin, Z., Yan, W., \& Zhang, J. (2017). Influence of cigarette smoking on osteonecrosis of the femoral head (ONFH): a systematic review and meta-analysis. Hip International, 27(5), 425-435. https://doi.org/10.5301/hipint.5000516

71. Sung, P. H., Yang, Y. H., Chiang, H. J., et al. (2018). Cardiovascular and cerebrovascular events are associated with nontraumatic osteonecrosis of the femoral head. Clinical Orthopaedics and Related Research, 476(4), 865-874. https://doi.org/10.1007/ s11999.0000000000000161

72. López Zúñiga, M. Á., Moreno-Moral, A., Ocaña-Granados, A., et al. (2021). High-dose corticosteroid pulse therapy increases the survival rate in COVID-19 patients at risk of hyper-inflammatory response. PLoS ONE. https://doi.org/10.1371/journal.pone.02439 64

73. Yang, L., Boyd, K., Kaste, S. C., Kamdem Kamdem, L., Rahija, R. J., \& Relling, M. V. (2009). A mouse model for glucocorticoid-induced osteonecrosis: effect of a steroid holiday. Journal of Orthopaedic Research, 27(2), 169-175. https://doi.org/10.1002/ jor.20733

74. Kang, P., Gao, H., Pei, F., Shen, B., Yang, J., \& Zhou, Z. (2010). Effects of an anticoagulant and a lipid-lowering agent on the prevention of steroid-induced osteonecrosis in rabbits. International Journal of Experimental Pathology, 91(3), 235-243. https://doi. org/10.1111/j.1365-2613.2010.00705.x
75. Wang, G. J., Cui, Q., \& Balian, G. (2000). The Nicolas Andry award. The pathogenesis and prevention of steroid-induced osteonecrosis. Clinical Orthopaedics and Related Research, 370(1), 295-310. https://doi.org/10.1097/00003086-200001000-00030

76. Ajmal, M., Matas, A. J., Kuskowski, M., \& Cheng, E. Y. (2009). Does statin usage reduce the risk of corticosteroid-related osteonecrosis in renal transplant population? Orthopaedic Clinics of North America, 40(2), 235-239. https://doi.org/10.1016/j.ocl. 2009.01.004

77. Glueck, C. J., Freiberg, R. A., \& Wang, P. (2020). Long-term anticoagulation prevents progression of stages I and II primary osteonecrosis of the hip in patients with familial thrombophilia. Orthopedics, 43(4), e208-e214. https://doi.org/10.3928/01477 447-20200404-06

78. Fu, W., Liu, B., Wang, B., \& Zhao, D. (2019). Early diagnosis and treatment of steroid-induced osteonecrosis of the femoral head. International Orthopaedics, 43(5), 1083-1087. https://doi.org/10. 1007/s00264-018-4011-y

79. Liu, B. Y., Yang, L., Wang, B. J., et al. (2017). Prevention for glucocorticoid-induced osteonecrosis of femoral head: a long-term clinical follow-up trail. Zhonghua Yi Xие Hui, 97(41), 3213-3218. https://doi.org/10.3760/cma.j.issn.0376-2491.2017.41.004

80. Patell, R., Chiasakul, T., Bauer, E., \& Zwicker, J. I. (2021). Pharmacologic thromboprophylaxis and thrombosis in hospitalized patients with COVID-19: a pooled analysis. Thrombosis and Haemostasis, 121(1), 76-85. https://doi.org/10.1055/s-0040-1721664

81. Sakamoto, M., Shimizu, K., Iida, S., Akita, T., Moriya, H., \& Nawata, Y. (1997). Osteonecrosis of the femoral head: a prospective study with MRI. Journal of Bone and Joint Surgery British, 79(2), 213-219.

82. Zhao, F. C., Li, Z. R., \& Guo, K. J. (2012). Clinical analysis of osteonecrosis of the femoral head induced by steroids. Orthopaedic Surgery, 4(1), 28-34. https://doi.org/10.1111/j.1757-7861. 2011.00163.x

83. Qi, X., \& Zeng, Y. (2015). Biomarkers and pharmaceutical strategies in steroid-induced osteonecrosis of the femoral head: a literature review. Journal of International Medical Research, 43(1), 3-8. https://doi.org/10.1177/0300060514554724

84. Chen, S., Cai, Q., Xu, Y., et al. (2021). Associations between glucocorticoids, antiphospholipid antibodies and femur head necrosis in patients with SLE: a directed acyclic graph-based multicentre study. Therapeutic Advances in Musculoskeletal Disease. https:// doi.org/10.1177/1759720X211002677

85. Ma, J., Guo, W., Li, Z., Wang, B., Li, S., \& Wang, P. (2017). Hip osteonecrosis is associated with increased plasma IL-33 level. Mediators of Inflammation. https://doi.org/10.1155/2017/17326 38

86. Huang, Z., Fu, F., Ye, H., et al. (2020). Chinese herbal Huo-Gu formula for the treatment of steroid-associated osteonecrosis of femoral head: a 14-year follow-up of convalescent SARS patients. Journal of Orthopaedic Translation, 23, 122-131. https://doi.org/ 10.1016/j.jot.2020.03.014

Publisher's Note Springer Nature remains neutral with regard to jurisdictional claims in published maps and institutional affiliations. 